\title{
4. EL PROCESO DE DESINSTITUCIONALIZACIÓN DE LOS HOSPITALES PSIQUIÁTRICOS:
}

En el cuestionario elaborado por la anterior Junta Directiva de la AEN, se incluyó un apartado sobre evaluación del Proceso de Reforma en las distintas CCAA. Nos ha parecido importante presentar el resumen de los datos aportados por cada una de ellas. En la tabla 44 mostramos la situación actual de los Hospitales Psiquiátricos.

Tabla 44. Hospitales Psiquiátricos

\begin{tabular}{|l|c|l|}
\hline COMUNIDAD AUTÓNOMA & HOSP. PSIQUIÁTRICO & DENOMINACIÓN \\
\hline ANDALUCIAA & $\mathrm{NO}$ & - \\
\hline ARAGÓN & $\mathrm{SI}$ & Centros de Rehabilitación Psicosocial \\
\hline ASTURIAS & $\mathrm{N} 0$ & - \\
\hline BALEARES & $\mathrm{SI}$ & Área de Salud Mental \\
\hline CANARIAS & $\mathrm{N} 0$ & - \\
\hline CANTABRIA & $\mathrm{SI}$ & Centro de Rehabilitación Psiquiátrica de Parayas y Padre Menni \\
\hline CASTILLA LA MANCHA & $\mathrm{SI}$ & Hospital Psiquiátrico de Alcohete \\
\hline CASTILLA LEÓN & $\mathrm{SI}$ & Unidad Residencial Psiquiátrica de referencia Regional \\
\hline CATALUÑA & $\mathrm{SI}$ & Hospital Monográfico \\
\hline EUSKADI & $\mathrm{SI}$ & Hospital Psiquiátrico y/o Monográfico \\
\hline EXTREMADURA & $\mathrm{SI}$ & Hospital Psiquiátrico \\
\hline GALICIA & $\mathrm{SI}$ & Hospital Psiquiátrico \\
\hline MADRID & $\mathrm{SI}$ & Hospital Psiquiátrico \\
\hline MELILLA & $\mathrm{N} 0$ & - \\
\hline MURCIA & $\mathrm{SI}$ & Unidades de: - Agudos, Subagudos y Rehabilitación - Crónicos y Psicogeriatría \\
\hline NAVARRA & $\mathrm{N} 0$ & - \\
\hline RIOJA & $\mathrm{SI}$ & Centro Asistencial Reina Sofía \\
\hline VALENCIA & $\mathrm{SI}$ & Hospital Psiquiátrico y Sanatorio de Santa Fe \\
\hline
\end{tabular}


Exponemos un resumen del proceso de desinstitucionalización en cada CCAA.

ANDALUCÍA: No existe Hospital Psiquiátrico. El proceso de desinstitucionalización, desmantelamiento y cierre de los 8 Hospitales Psiquiátricos que existían se realizó entre los años 1985 y 1999. Al mismo tiempo que se cerraban los Hospitales, se abrían Comunidades Terapéuticas y Casas Hogares.

ARAGÓN: Los Hospitales Psiquiátricos se denominan Centros de Rehabilitación Psicosocial. Existen en las tres provincias de Aragón y se componen de Unidad de Media Estancia, Unidad de Larga Estancia y Unidad Gerontopsiquiátrica (actualmente sólo existe en Zaragoza, en Huesca y Teruel están en obras).

Los pacientes de los Hospitales Psiquiátricos que fueron externalizados se enviaron a Residencias, Centros de Deficientes, Recursos Residenciales protegidos (pisos, casa, hogares) y Familias.

ASTURIAS: No existe Hospital Psiquiátrico. En los terrenos del antiguo Hospital Psiquiátrico existen actualmente la Unidad Residencial, Unidad de Psicogeriatría y Unidad de Rehabilitación.

Previo al proceso de derribo de los edificios del antiguo Hospital Psiquiátrico de Oviedo en el año 2003, se derivaron pacientes de las Unidades Residenciales a establecimientos Residenciales en toda Asturias.

BALEARES: El Hospital Psiquiátrico se denomina Área de Salud Mental del Complejo Hospitalario de Mallorca. La gestión la realiza la empresa pública con gestión privada, Gestión Sanitaria de Mallorca (GESMA). En estos momentos, en el recinto del Hospital Psiquiátrico se encuentran el Psicogeriátrico (58 plazas), Unidad de Larga Estancia y Residencia Mixta (48 plazas), Pabellón Lluerna (24 plazas) y Unidad de Hospitalización Breve (30 camas) con Servicio de Urgencias Psiquiátricas

El proceso de desinstitucionalización se inició hace un año aunque desde hace varios años ya no ingresan pacientes a las Unidades de Larga Estancia.

Los pacientes (155) fueron evaluados entre 2000-2001 y han sido externalizados a Residencias, Recursos residenciales protegidos (pisos, casas, hogares) y Familias.

CANARIAS: No existe Hospital Psiquiátrico. Las distintas Unidades de los Hospitales Psiquiátricos se han ido transformando en Unidades de Rehabilitación Activas. A la vez se han ido creado alternativas residenciales comunitarias.

CANTABRIA: Los Hospitales Psiquiátricos se denominan Centro de Rehabilitación Psiquiátrica de Parayas (depende administrativamente del Servicio Cántabro de Salud) y Padre Menni (Concertado). Tienen en total 443 plazas.

CASTILLA LEÓN: Existe un Hospital Psiquiátrico que se denomina Unidad Residencial Psiquiátrica de referencia Regional con 50 camas.

CASTILLA LA MANCHA: El único que en estos momentos existe como Hospital Psiquiátrico es el de Alcohete. Los demás Hospitales Psiquiátricos que existían (6 en total, con 450 plazas) están en proceso de reconversión en Unidades de Media Estancia, Unidades Residenciales y Residencias Sociosanitarias, excepto el de Ciudad Real que es Unidad de Media Estancia y Residencia Psicogeriátrica desde Diciembre de 1998. 
El Hospital Psiquiátrico de Albacete lo cerrarán en el 2004.

Los pacientes después de un proceso de evaluación, han sido externalizados a Residencias, Centros de deficientes, Recursos residenciales protegidos, Familia, etc.

CATALUÑA: Existe el Hospital Psiquiátrico, pero se denomina Hospital Monográfico. Hay 7 centros en Cataluña que dependen administrativamente de Sanidad. El total de plazas que tienen es de 2875.

El Proceso de Desinstitucionalización está actualmente en curso. Se está realizando el estudio de los pacientes de Larga Estancia para su externalización y reubicación en otros dispositivos. Los pacientes que ya se han externalizado han sido destinados a Residencias, Familia y Unidades Polivalentes.

El acuerdo de reconversión de los Hospitales Monográficos plantea la creación de Unidades de Agudos, Unidades Polivalentes: De alta dependencia, de Patología Dual, Unidad de Hospitalización Psiquiátrica Penitenciaria y una Unidad de Alta Seguridad.

EUSKADI: Existen Hospitales Psiquiátricos. El Servicio Vasco de Salud (Osakidetza) tiene 4 Hospitales Psiquiátricos (Álava, Bermeo, Zaldibar y Zamudio) con un total de 800 plazas. Además existen tres Hospitales Psiquiátricos(Aita Menni) con plazas concertadas.

El proceso de desinstitucionalización ha sido progresivo y el destino de los pacientes externalizados ha sido en residencias, recursos residenciales protegidos (pisos, casa, hogares) y familia.

EXTREMADURA: Existen 2 hospitales psiquiátricos, uno en Badajoz y otro en Cáceres. Tienen un total de 835 plazas y dependen administrativamente de las Diputaciones Provinciales. En la actualidad están en un proceso de integración en el Servicio Extremeño de Salud.

En el año 1996, se aprobó el Proyecto Augusta para externalización de pacientes. Hasta estos momentos se han externalizado a pisos a 49 pacientes.

GALICIA: Existe un hospital psiquiátrico dependiente de la Diputación de Lugo que actualmente está en proceso de transferencia a la Comunidad Autónoma.

MADRID: Existen tres hospitales psiquiátricos: Centro San Juan de Dios, Centro Benito Menni y Hospital Psiquiátrico. Dependen administrativamente de la Consejería de Sanidad y de la Consejería de Servicios Sociales. El número de plazas totales es de 1712. Los hospitales cuentan con Unidad de Hospitalización Breve (123 camas de agudos), Unidad de Media Estancia (131), Unidad de Larga Estancia (524), Psicogeriatría (498) y Centros de Deficientes Mentales (426).

El programa de desinstitucionalización se llevó a cabo en los años 80. Actualmente no existe.

MELILLA: No existe hospital psiquiátrico.

MURCIA: Existe hospital psiquiátrico. Tiene varias unidades: 2 Unidades de agudos con un total de 52 camas, además una Unidad denominada de Sub-agudos con 48 camas y una Unidad de Rehabilitación con 60 plazas. Administrativamente depende del Servicio Murciano de Salud. 
La Consejería de Bienestar Social del Gobierno Murciano tiene el Instituto de Servicios Sociales (ISSORN) y de él dependen la Unidad de Crónicos Luis Valenciano (50 camas), la Unidad de Psicogeriatría Javier Asturiano (54 camas) y otra Unidad de Psicogeriatría que es una residencia para la tercera edad (30 camas).

NAVARRA: No existe hospital psiquiátrico.

Entre 1986 y 1991 se trasladaron varios pacientes del Hospital Psiquiátrico a otros recursos: 73 a Residencias, 48 a residencias protegidas, 18 fueron con su familia y 20 pacientes quedaros sin ubicación. Por otra parte, se realizó la transferencia de una Residencia de Deficientes Mentales (donde estaban 100 pacientes) desde la Consejería de Sanidad a la Consejería de Bienestar Social.

En 1998, se aprueba por Decreto la transformación del Hospital Psiquiátrico en Centro Psicogeriátrico.

RIOJA: El Hospital Psiquiátrico se denomina Centro Asistencial Reina Sofía. Depende administrativamente del Servicio Riojano de Salud y de la Consejería de Bienestar Social. Tiene aproximadamente 200 plazas.

El proceso de desinstitucionalización de los pacientes del Hospital Psiquiátrico se iniciará a partir del 2004. Desde hace 10 años, para ingresar en el hospital han de pasar por una comisión constituida por diferentes psiquiatras. En la actualidad se está en proceso de evaluación de pacientes para poder remitirlos a Residencias, Recursos residenciales protegidos, Centros de deficientes, Familia, etc.

El Hospital Psiquiátrico cuenta actualmente con diferentes pabellones: Larga Estancia, Media Estancia, Residencia Geriátrica y Residencia de Minusválidos Psíquicos.

VALENCIA: Existen dos hospitales psiquiátricos: Padre Jofré en Bétera (Valencia) y el Sanatorio de Santa Faz (Alicante). La dependencia administrativa es de las Diputaciones provinciales. No se sabe el número de plazas de los Hospitales. Actualmente no ingresan prácticamente pacientes. 